\title{
Efficacy of Lumboperitoneal Shunting in Patients with Normal Pressure Hydrocephalus
}

\author{
Mehmet Akif BAYAR ${ }^{1}$, Ayhan TEKINER ${ }^{1}$, Haydar CELIK ${ }^{1}$, Ali YILMAZ², Guner MENEKSE ${ }^{1}$, Timur YILDIRIM², \\ Fatih ALAGOZ3 ${ }^{3}$, Yahya GUVENC ${ }^{4}$, Yavuz ERDEM ${ }^{1}$ \\ ${ }^{1}$ Ankara Training and Research Hospital, Department of Neurosurgery, Ankara, Turkey \\ ${ }^{2}$ Ordu University, Faculty of Medicine, Department of Neurosurgery, Ordu, Turkey \\ ${ }^{3}$ Ankara Numune Training and Research Hospital, Department of Neurosurgery, Ankara, Turkey \\ ${ }^{4}$ Marmara University Faculty of Medicine, Department of Neurosurgery, Istanbul, Turkey
}

\section{ABSTRACT}

AIM: To evaluate the effectiveness of lumboperitoneal (LP) shunt procedures in the treatment of normal pressure hydrocephalus (NPH).

MATERIAL and METHODS: A medical record review was conducted to obtain demographic, clinical, laboratory data, as well as pre-, intra, -postoperative details of 65 patients who underwent LP shunt surgery for NPH between January 1, 2001 and January 1, 2014 in the Neurosurgery Clinics of Ankara Training and Research Hospital and Ankara Numune Hospital.

RESULTS: At the $3^{\text {rd }}$ month after LP shunt surgery, headache was resolved in almost all patients. At the end of first year, while statistically significant improvements were noted in the Modified Rankin Scale Scores and Mini-Mental State Examination Scores, gait disturbance, urinary incontinence and cognitive functions were improved by $86 \%, 72 \%$ and $65 \%$ of the patients, respectively.

CONCLUSION: LP shunt surgery is associated with a lower rate of complication in comparison to ventriculoperitoneal shunt surgery and is an effective procedure in the treatment of $\mathrm{NPH}$.

KEYWORDS: Lumboperitoneal shunt, Normal pressure hydrocephalus, Cerebro-spinal fluid

\section{INTRODUCTION}

$\mathrm{N}$ ormal pressure hydrocephalus (NPH) is a syndrome first defined in 1965 by Hakim and Adams. This syndrome is a significant neurological condition that occurs predominantly in elderly people and its clinical presentation consists of a triad of major symptoms, including gait disturbance, dementia and urinary incontinence (12).

Surgical treatment of NPH typically includes the diversion of cerebro-spinal fluid (CSF) into the peritoneum, pleura or atrium. While ventriculoperitoneal shunt (VPS) surgery has historically been widely performed, today, minimally invasive surgical procedures such as lumboperitoneal shunt (LPS) have become the surgical treatment of choice (2). The LPS surgery is one of the common effective treatment modalities used in the surgical management of communicating hydrocephalus, pseudotumor cerebri, CSF fistula and normal pressure hydrocephalus (NPH)(13).

Its relative ease to perform and shorter surgery time, lack of risk for intracranial complications, including intracerebral hemorrhages, seizures or shunt dysfunction, lower risk of shunt infections and lethal complications all make LPS a favorable surgical treatment modality as compared to VPS surgery. Ventricular catheter placement may be challenging in patients with small ventricles, even though the current use of navigation has provided convenience in placing a shunt catheter and higher success rates. Therefore, its simplicity 
may be the motive to prefer LPS surgery in patients with small ventricles $(1,4,19,20,22)$. LPS systems are constituted of 3 main components: a lumbar catheter, a valve, and a peritoneal catheter (18). A medium pressure, flow control shunt system [medium pressure flow control valve (Medtronic $®)$ ] was used in all patients who were included in our study.

LPS surgery provided significantly high rates of clinical improvement and an increased quality of life and these outcomes were comparable to that of VPS procedures (3). This study aimed at reviewing the demographic characteristics, mortality and morbidity rates, complications, treatment response and prognosis in 65 cases of NPH who had been treated with a flow and pressure control LPS implantation in our clinics.

\section{MATERIAL and METHODS}

The clinical data of 65 patients with NPH treated with LPS surgery in the Department of Neurosurgery of Ankara Training and Research Hospital and the Department of Neurosurgery of Ankara Numune Training and Research Hospital over a 13-year period were retrospectively analyzed. Data were obtained from medical charts, follow-up examinations and neuroimaging studies of the patients. Demographic characteristics, including age, gender, clinical symptoms, opening pressure, neuroimaging, complications, pre- and postoperative outcome scale scores (Modified Rankin Score (mRS) and Mini-Mental State Examination (MMSE) score) were assessed. There were 65 cases of $\mathrm{NPH}$, of whom 30 $(46.2 \%)$ were female and 35 (53.8\%) were male. The mean age was $64.4 \pm 9.5$ (Min=38, Max=78) years.

\section{Clinical Manifestation}

Cognitive dysfunction was the most common symptom and it was present in $87 \%$ of the patients. This symptom was followed by gait disturbance $(80 \%)$ and urinary symptoms $(62 \%)$. The classical triad of the syndrome was detected in only $40 \%$ of patients. In addition to these symptoms, headache was present in $54 \%$ of the patients. Ophthalmoscopic examination was routinely performed to detect papilledema and to exclude a possible diagnosis of pseudotumor cerebri.

\section{Imaging Examination}

Each patient routinely underwent a computed tomography (CT) scan of the brain and magnetic resonance imaging (MRI) for diagnostic and follow-up purposes, and to exclude possible intracranial mass lesions that may be considered in the differential diagnosis and to assess ventricular sizes and CSF circulation.

\section{Patient Selection}

A routine lumbar puncture was performed in all patients who presented with clinical and radiological symptoms suggesting $\mathrm{NPH}$. Patients were considered as NPH if the opening pressure ranged from 70 to $245 \mathrm{~mm} \mathrm{H}_{2} \mathrm{O}$. Lumbar punctures were repeated during 3 days and $30 \mathrm{ml}$ of CSF was drained each time. Patients were considered as candidates for LPS surgery, in the event that improvements were observed in their clinical findings following this procedure. Biochemical and microbiological analyses were performed on CSF samples obtained via lumbar puncture. Glucose, protein and white blood cell (WBC) values were the parameters to be considered in surgical treatment planning. LPS surgery was not performed in the event that CSF analyses showed a WBC count of $\geq 60$ / $\mathrm{mm}^{3}$ and a protein level of $\geq 1000 \mathrm{mg} / \mathrm{L}$.

\section{Surgical Technique}

All patients underwent general anesthesia and endotracheal intubation. They were typically placed in a lateral decubitus position. C-arm fluoroscopy was used for the detection of $L 4-5$ or L3-4 spinal level. After preparation of the skin and draping of the surgical field, three incisions were made, including a longitudinal incision in the posterior midline, a 4-6 cm flank incision and a medial ventral incision. Approximately 10-15 $\mathrm{cm}$ of the lumbar catheter was inserted into the subarachnoid space using a Tuohy needle, and this procedure was confirmed via CSF flow. To place the valve, a flank incision was used. A direct approach to the peritoneum was usually used with a medial ventral incision. Dissection of the fasciae and muscle layers was performed and a peritoneal catheter was inserted and tunneled between the lumbar and medial ventral incision. The flow-pressure-controlled valve was anchored with the use of silk sutures. Flow from the distal end of the shunt system should be examined before placement into the peritoneal cavity. Closure was carried out in the standard fashion.

\section{Follow-up and Outcome Measures}

In the follow up period, neurological examination was repeated at 3, 6 and 12 months. The mRS was used to measure the degree of disability or dependence in the daily activities and the MMSE was used to measure cognitive functions of our patients. These measurements were performed at baseline and at 12 months after surgery.

\section{Statistical Analysis}

Statistical analyses were performed using the SPSS 15.0 software for Windows (SPSS Inc, Chicago, IL, USA). P values less than 0.05 were considered as statistically significant. The Wilcoxon Signed Ranks Test was used for intergroup comparisons.

\section{- RESULTS}

At the end of $3^{\text {rd }}$ month after LPS surgery, the complaint of headache was resolved in all patients. At the end of the first year, improvements were observed in gait disorder, urinary incontinence and cognitive functions in $86 \%, 72 \%$ and $65 \%$ of the patients, respectively.

During the early post-operative period, two patients developed a small amount of subcutaneous CSF accumulation in the lumbar region and one patient developed a small amount of subcutaneous CSF accumulation in the abdominal region. These patients were managed in a conservative fashion and they did not require shunt revision surgery. In our study, no patient developed any neurogenic or intra-abdominal complication. Only two cases of shunt infection (3\%) were 
observed in this study. LPSs were removed in these two patients and they were treated with an appropriate antibiotic regimen. These patients underwent VPS surgery following the resolution of the signs and symptoms of the infection. At the end of the first year, 3 patients were reported to have died due to systemic diseases, independently from NPH. The patients who died or underwent revision and VPS surgery were not included in the analysis at the end of the first year.

The MMSE and mRS assessments of the clinical response of 65 patients, who were included in the study, revealed that this surgical treatment modality was associated with statistically significant improvements in the test scores $(p<0.001)$ (Figures 1, 2).

\section{DISCUSSION}

Normal pressure hydrocephalus is characterized by gait apraxia, urinary incontinence and cognitive dysfunction (Hakim-Adams syndrome). The triad of Hakim-Adams syndrome is present in almost $50 \%$ of cases, however, only one or a combination of two symptoms should be considered for investigation and diagnosis (17). The possible mechanisms of $\mathrm{NPH}$ are still not completely understood. A commonly accepted theory is low venous compliance in the basal ganglia, thalamus and usually frontal lobe. Manometric studies have demonstrated the affecting circulation and absorption of CSF in the subarachnoid granulations. Moreover, there are several diseases that present the similar symptoms as the classic NPH triad (17).

The aim of treatment is to restore the patient's functional capacity. Up till now, diagnostic tests have not been sufficient for diagnosis and predicting postoperative outcome. Early onset of gait apraxia and onset of symptoms for less than six months are the common indicators of surgical treatment. VPS, endoscopic third ventriculostomy and LPS are the main treatment options. Nevertheless, there are questions about which modality is effective and what type of shunt valve should be implemented (24).

LPS surgery is a safe, effective treatment modality, is, still upto-date and is used to treat a number of diseases such as pseudotumor cerebri, post-operative pseudomeningocele, CSF fistula, NPH and other forms of communicating hydrocephalus $(5,6,19)$. The series of cases of LPS surgery in the medical literature have usually included patients from adult age groups and the most common underlying pathologies were NPH secondary to subarachnoid hemorrhage, idiopathic $\mathrm{NPH}$ and hydrocephalus secondary to head trauma $(1,8)$. Our study included patients from adult age groups who were diagnosed with NPH.

In our study, the decision to treat NPH patients with LPS surgery was made on the basis of symptoms, neurological examination, the findings on the imaging studies such as $\mathrm{MRI}$ and CT of the patients and their response to spinal tap. A medium pressure, flow control shunt system was used in all patients who underwent surgery. While CT scans were preferred as the imaging modality for the follow-up during the early postoperative period, MRI was preferred for long-term follow-up. LPS surgery may be associated with neurological and non-neurological complications. LPS-related nonneurological complications may occur anywhere between the spinal region and peritoneal region where the abdominal segment of the catheter is implanted, as well as in the thoracic cavity, intra-abdominal solid organs or in any hollow organs such as intestines and ureters $(11,14,23)$. Although LPS surgery has provided favorable outcomes, occlusion has been reported at a rate of $7 \%$ and catheter migration, pseudocysts and infections have been reported at a rate up to $14 \%$, in the medical literature (14). In our study, no neurological complication occurred in any case. Only 3\% of the patients $(n=2)$ developed shunt infections and this rate was lower than the infection rate reported in the medical literature (3).

One of the major advantages of LPS surgery over VPS is that it lacks the risk of intracranial complications such intracerebral hemorrhages, seizures or shunt malpositioning, and this advantage is one of the main factors that make it the preferred surgical treatment at an increasing rate $(1,19,20)$. VPS surgery

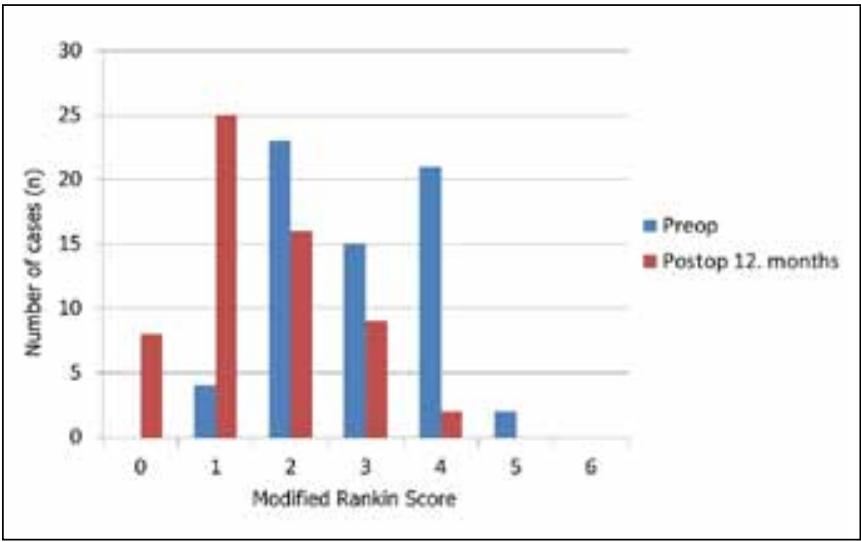

Figure 1: Changes in the scores of the modified Rankin Scale (mRS) one year after the shunting compared with those recorded before shunting.

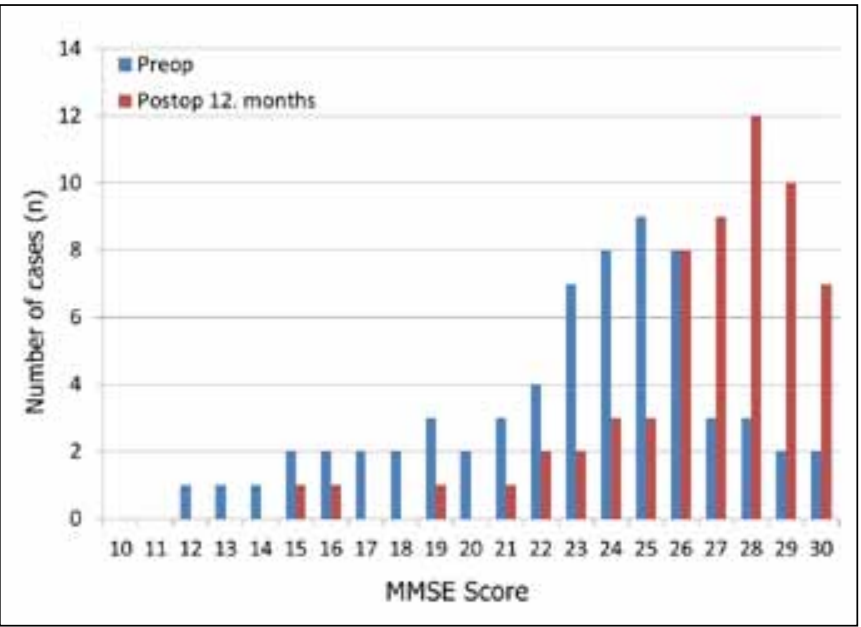

Figure 2: Changes in the scores of the Mini-Mental State Examination (MMSE) one year after the shunting compared with those recorded before shunting. 
has been reported to be associated with an obstruction risk of $58 \%$ and the rate of epilepsy associated with cortical damage due to ventricular catheter insertion has been found to be considerable and to range from $9.4 \%$ to $24 \%(7,21)$. A serious complication at such a high rate may be avoided by LPS surgery.

Although favorable outcomes have been reported, occlusion and shunt dysfunction may occur following LPS surgery and a shunt revision surgery may be required. Furthermore, LPS surgery should be avoided in the presence of tonsillar herniation, infections and arachnoiditis (11).

In a study conducted by Klinge et al., the rate of treatment response was reported to be $69 \%$ to $84 \%$ among NPH patients who underwent LPS surgery, while the need for a revision surgery was found to be $15 \%$ in patients who developed complications (15). Moreover, in a series of 33 cases published by Bloch et al., shunt dysfunction and the need for a revision surgery was reported as $27 \%$. (3). In a study of 195 patients who underwent LPS surgery, Duther et al. reported that shunt dysfunction occurred in 28 patients (14.3\%) out of 47 patients who developed complications (9). In a study conducted by Eggenberger et al., shunt dysfunction due to shunt occlusion was reported as the most common complication of LPS surgery in patients with pseudotumor cerebri (10). In our study, shunt dysfunction did not occur during the first year of follow-up period, however LPS systems were removed in the patients who developed shunt infections and these two patients underwent VPS surgery following the treatment with an appropriate antibiotic regimen.

Subcutaneous infections, gallbladder perforation, pneumothorax, mesenteric inflammation, intestinal obstruction, the migration of the peritoneal catheter into the scrotum, umbilicus, rectum or vagina, intestinal perforation, abdominal cyst formation, inguinal hernia, migration into the liver and liver abscess are the other LPS-related complications reported in the literature $(1,6,11,23,24)$. In our patients, no such serious peritoneal catheter-related complications were observed. Only a limited amount of subcutaneous fluid accumulation occurred in 2 patients during the early postoperative period but revision surgery was not required in these patients.

Even though rates of shunt catheter-related malpositioning and malfunction have been reported to be higher in LPS surgery, the risk of mortality associated with LPS surgery was lower than with VPS surgery (3). When considering the pros and cons, LPS surgery is associated with a lower rate of complications and it may be considered as a minimally invasive treatment modality with better outcomes.

When compared to VPS, lower rates of overall mortality have been reported in the series of patients who underwent LPS surgery in the literature $(16,25)$. No case of surgery-related death occurred in our patients who underwent LPS surgery. Death from systemic disorders occurred in 3 patients $(4.6 \%)$ during the postoperative period.

\section{- CONCLUSION}

LPS surgery is a safe, minimally invasive treatment modality with lower rates of complication, in comparison to VPS surgery and is associated with successful outcomes. LPS surgery is an effective surgical modality and may be used as an alternative to VPS surgery in the treatment of NPH patients.

\section{- REFERENCES}

1. Aoki N: Lumboperitoneal shunt: Clinical applications, complications and comparisons with ventriculoperitoneal shunt. Neurosurgery 26: 998-1004; 1990

2. Bergsneider $M$, Black $P M$, Klinge $P$, Marmarou $A$, Relkin $\mathrm{N}$ : Surgical management of idiopathic normal-pressure hydrocephalus. Neurosurgery 57(3 Suppl): 29-39; discussion ii-v. Review, 2005

3. Bloch O, McDermott MW: Lumboperitoneal shunts for the treatment of normal pressure hydrocephalus. J Clin Neurosci 19: 1107-1111, 2012

4. Brazis PW: Clinical review: The surgical treatment of idiopathic pseudotumour cerebri (idiopathic intracranial hypertension). Cephalalgia 28: 1361-1373, 2008

5. Bret P, Hor F, Huppert J, Lapras C, Fischer G: Treatment of cerebrospinal fluid rhinorrhea by percutaneous lumboperitoneal shunting: Review of 15 cases. Neurosurgery 16: 44-47, 1985

6. Chumas PD, Kulkarni AV, Drake JM, Hoffman HJ, Humphreys RP, Rutka JT: Lumboperitoneal shunting. A retrospective study in pediatric population. Neurosurgery 32: 376-383, 1993

7. Copeland G, Foy P, Shaw M: The incidence of epilepsy after ventricular shunting operations. Surg Neurol 17:279-281, 1982

8. Duthell R, Cristophe N, Fotso MJ, Beauchesne P, Jacques B: Lumboperitoneal shunting. In: Schmidek Sweet (ed). Operative Neurosurgical Techniques, Indications, Methods and Results. Philadelphia: WB Saunders, 2000: 604-607

9. Duther R, Nuti C: Complications of lumboperitoneal shunts. A retrospective study of series of 195 patients. Neurochirurgie 42: 83-89, 1996

10. Eggenberger RE, Miller NR, Vitake S: Lumboperitoneal shunt for treatment of pseudotumor cerebri. Neurology 46:15241530, 1996

11. Fukamachi A, Wada H, Toyoda O, Wakao T, Kawafuchi J: Migration or extrusion of shunt catheters. Acta Neurochir 64: 160-166, 1982

12. Hakim S, Adams RD: The special clinical problem of symptomatic hydrocephalus with normal cerebrospinal fluid pressure. Observations on cerebrospinal fluid hydrodynamics. J Neurol Sci 2: 307-327, 1965

13. Karabatsou K, Quigley G, Buxton N, Foy P, Mallucci C: Lumboperitoneal shunts: Are the complications acceptable? Acta Neurochir (Wien) 146: 1193-1197, 2004

14. Kelman SE, Sergot RC, Cioffi GA, Savino PJ, Bosley TM, Elman MJ: Modified optic nerve sheath decompression in patients with functioning lumboperitoneal shunt and progressive visual loss. Ophthalmology 98: 1449-1453,1991 
15. Klinge P, Hellstrom P, Tans J, Wikkelsø C: European iNPH Multicentre Study Group: One-year outcome in the European multicentre study on iNPH. Acta Neurol Scand 126:145-153, 2012

16. Lamprect D, Schoeman J, Donald P, Hartzenberg $H$ : Ventriculoperitoneal shunting in childhood tuberculous meningitis. Br J Neurosurg 15: 119-125; 2001

17. Oliveira MF, Reis RC, Trindade EM, Pinto FC: Evidences in the treatment of idiopathic normal pressure hydrocephalus. Rev Assoc Med Bras 61: 258-262, 2015

18. Post EM: Shunt systems. Wilkins RH, Rengachary SS (ed), Neurosurgery, 2nd ed. New York: McGraw-Hill, 1996: 36453653

19. Rekate $H$, Wallace D: Lumboperitoneal shunts in children. Pediatr Neurosurg 38: 41-46, 2003

20. Satow T, Motoyama Y, Yamazoe N, Isaka F, Higuchi K, Nabeshima S: Migration of a lumboperitoneal shunt catheter into the spinal canal-Case report. Neurol Med Chir (Tokyo) 41: 97-99, 2001
21. Sood S, Lokuketagoda J, Ham SD: Periventricular rigidity in long-term shunt-treated hydrocephalus. J Neurosurg 102: 146-149, 2005

22. Toma AK, Dherijha M, Kitchen ND, Watkins LD: Use of lumboperitoneal shunts with the Strata NSC valve: A singlecenter experience. J Neurosurg 113: 1304-1308, 2010

23. Touho H, Nakauchi M, Tasawa T, Nakagawa J, Karasawa J: intra hepatic migration of a peritoneal shunt catheter: Case report. Neurosurgery 21: 258-259, 1987

24. Wang VY, Barbaro NM, Lawton MT, Kunwar S, Gupta N, Mcdermott MW: Complications of lumboperitoneal shunts. Neurosurgery 60: 1045-1048, 2007

25. Yadav YR, Sanjay P, Raina VK, Singh M: Lumboperitoneal shunts: Review of 409 cases. Neurol India 52: 188-190, 2004 